\title{
E3 ubiquitin ligase HECTD2 mediates melanoma progression and immune evasion
}

Eleonora Ottina (D) ${ }^{1}$, Veera Panova (iD ${ }^{1}$, Laura Doglio ${ }^{1}$, Anastasiya Kazachenka ${ }^{1}$, Georgina Cornish ${ }^{1}$, Joanna Kirkpatrick ${ }^{2}$, Jan Attig (iD ${ }^{1}$, George R. Young (iD ${ }^{3}$, Kevin Litchfield (iD) ${ }^{4}$, Tom Lesluyes (iD) ${ }^{5}$, Peter Van Loo (i) ${ }^{5}$, Charles Swanton (iD) ${ }^{4}$ James MacRae (iD) ${ }^{2}$, Thomas Tüting (iD) ${ }^{6}$ and George Kassiotis (iD ${ }^{1,7 凶}$

(c) The Author(s) 2021, corrected publication 2021

The ubiquitin-proteasome system maintains protein homoeostasis, underpins the cell cycle, and is dysregulated in cancer. However, the role of individual E3 ubiquitin ligases, which mediate the final step in ubiquitin-mediated proteolysis, remains incompletely understood. Identified through screening for cancer-specific endogenous retroviral transcripts, we show that the little-studied E3 ubiquitin ligase HECTD2 exerts dominant control of tumour progression in melanoma. HECTD2 cell autonomously drives the proliferation of human and murine melanoma cells by accelerating the cell cycle. HECTD2 additionally regulates cancer cell production of immune mediators, initiating multiple immune suppressive pathways, which include the cyclooxygenase 2 (COX2) pathway. Accordingly, higher HECTD2 expression is associated with weaker anti-tumour immunity and unfavourable outcome of PD-1 blockade in human melanoma and counteracts immunity against a model tumour antigen in murine melanoma. This central, multifaceted role of HECTD2 in cancer cell-autonomous proliferation and in immune evasion may provide a single target for a multipronged therapy of melanoma.

Oncogene (2021) 40:5567-5578; https://doi.org/10.1038/s41388-021-01885-4

\section{INTRODUCTION}

Cancer initiation and progression depends on the balance of multiple pro-tumour and anti-tumour processes. These include cancer cell-intrinsic mechanisms regulating cell proliferation, survival and migration [1, 2], and extrinsic factors, such as stromal cells in the tumour microenvironment and the anti-tumour immune response [3-6]. These processes are connected and a clear link between cancer cell-intrinsic genetic programs and antitumour immunity is beginning to emerge [7-9]. For example, defects in DNA damage repair and concomitant increase in mutation load or reactivation of endogenous retroviruses are thought to contribute to tumour immunogenicity by providing tumour-associated antigens [9-12]. In other instances, cancer cell cycle and proliferation programmes are under the control of transcription factors that also regulate the production of immune mediators, with NF-KB being a prime example [9]. Thus, cancer cell-intrinsic properties shape anti-tumour immunity, which in turn influences tumour evolution. However, the full extent of such bidirectional communication is incompletely understood and its outcome specific to the type of cancer [13].

Skin cutaneous melanoma (SKCM) is one of the most aggressive cancer types with increasing incidence worldwide [14]. It exhibits the highest load of somatic mutations among all cancer types [15] and can be inhibited by natural anti-tumour immunity and immunotherapy $[16,17]$. Although intensely studied, the precise mechanisms underlying melanoma progression and evasion of natural and immunotherapy-induced immunity remain unclear. We recently examined the transcriptional activity of endogenous retroviruses in cancer and identified a novel transcript spanning the HECTD2 locus and expressed uniquely in melanoma [18]. The novel transcript, [HECTD2-AS]HERVH-2, is transcribed antisense to HECTD2 and uses a human endogenous retrovirus $\mathrm{H}(H E R V H)$ provirus as terminal exon and polyadenylation signal [18]. Antisense transcription is mutually exclusive of sense transcription and strongly associated with a better prognosis of primary SKCM and uveal melanoma (UVM) [18]. These findings suggested a functional tumour-promoting role for HECTD2 in melanoma progression, which, however, remained unexplored.

HECTD2 is one of 28 members of the homologous to E6AP Cterminus (HECT) E3 ubiquitin ligases in humans [19]. Despite being essential for diverse cellular processes, most members of HECT E3 ubiquitin ligases, and HECTD2 in particular, are relatively understudied. HECTD2 was first identified as a candidate susceptibility gene for prion and Alzheimer's diseases [20,21], but its substrates or mode of action remained unknown. A recent study identified PIAS1 (protein inhibitor of activated STAT-1) as a direct HECTD2 target [22]. PIAS1 is an E3 SUMO-protein ligase that negatively regulates key inflammatory pathways, including NF-KB and its targeting for degradation by HECTD2 is required for maximal NF$K B$ activation and innate immunity in the lung [22]. The role of

\footnotetext{
${ }^{1}$ Retroviral Immunology Laboratory, The Francis Crick Institute, London, UK. ${ }^{2}$ Proteomics STP, The Francis Crick Institute, London, UK. ${ }^{3}$ Retrovirus-Host Interactions Laboratory, The Francis Crick Institute, London, UK. ${ }^{4}$ Cancer Evolution and Genome Instability Laboratory, The Francis Crick Institute, London, UK. ${ }^{5}$ Cancer Genomics Laboratory, The Francis Crick Institute, London, UK. ${ }^{6}$ Laboratory of Experimental Dermatology, Department of Dermatology, University of Magdeburg, Magdeburg, Germany. ${ }^{7}$ Department of Infectious

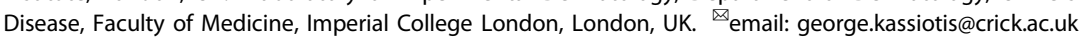

Received: 11 January 2021 Revised: 14 May 2021 Accepted: 1 June 2021

Published online: 18 June 2021 
HECTD2 in cancer has not yet been examined. However, two recent studies have suggested a possible involvement [23, 24]. HECTD2 was identified as a candidate driver gene in neuroblastoma [24] or as a potential target of miR-221, which promotes androgen-independent growth of prostate cancer cell lines [23], thus indicating an anti-proliferative role for HECTD2 in this cancer type.

Here, we directly investigated the possible involvement of HECTD2 in melanoma, suggested by the discovery of the melanoma-specific [HECTD2-AS]HERVH-2 antisense transcript and its association with better prognosis [18] and uncovered an unexpectedly central role.

\section{RESULTS}

\section{HECTD2 expression defines transcriptional clusters in SKCM}

To investigate the basis for the association of HECTD2 expression with SKCM survival, we first examined whether it marked disease subsets. Sample distance analysis of 443 SKCM samples from the cancer genome atlas [25] (TCGA) identified 4 distinct clusters based on the expression of all genes (Fig. 1a). Notably, HECTD2 expression was second only to IncRNA LINC02503 as the top gene responsible for the observed clustering (Fig. 1a). Clusters characterised by high HECTD2 expression ( 2 and 4$)$ were enriched for metastatic disease, where HECTD2 expression is higher [18] (Fig. 1b). The TCGA-defined [25] 'keratin' subtype was underrepresented in clusters 2 and 4, whereas the 'melanocyte inducing transcription factor (MITF)-low' subtype was almost exclusive to these high HECTD2-expressing clusters (Fig. 1b). For samples where 'immune landscape' annotation was available [26], the 'inflammatory' subtype was underrepresented in clusters 2 and 4, whereas the 'IFN- $\gamma$ dominant' and 'lymphocyte depleted' subsets enriched (Fig. 1b). Thus, clusters identified by sample transcriptional distance and characterised by distinct HECTD2 expression levels correlated well with previously identified molecular and immune subtypes $[25,26]$.

To further probe the potential influence of HECTD2 expression on the observed transcriptional profiles, we filtered genes differentially expressed between subsets with high ( $\geq 2$ transcripts per million, TPM) or low expression of HECTD2 (Fig. 1c). This analysis identified over 6000 differentially expressed genes $(\geq 2$ fold, $q \leq 0.05$ ) in primary SKCM, metastatic SKCM and UVM separately, the majority of which (5166) were differentially expressed in all three conditions (Fig. 1c). Moreover, nearly all of these genes were upregulated in high HECTD2-expressing samples in all three conditions (Fig. 1c). Lastly, functional enrichment analysis of the top 1000 genes upregulated in HECTD2-expressing samples identified two major pathways, protein modification (including ubiquitin-dependent protein degradation) and cell cycle (Fig. 1d). Together, these data raised the possibility that HECTD2 expression was part, if not a driver, of the extensive transcriptional dichotomy of melanoma.

\section{HECTD2 cell-autonomously promotes melanoma cell proliferation}

Whereas induction of genes involved in protein modification and degradation was expected in samples expressing high levels of HECTD2, induction of cell cycle-related genes was not. Despite its essential role in maximal NF-KB activation [22], HECTD2 has not been previously associated with the cell cycle and the only currently available data suggest an anti-proliferative role for HECTD2 in androgen-independent growth of the LNCaP prostate cancer cell line [23]. We, therefore, investigated if the apparent induction of cell cycle genes in melanoma biopsies with high HECTD2 expression could be due to a cell-autonomous proliferative effect of HECTD2. The upregulated genes in melanoma biopsies covered all stages of the cell cycle but were particularly enriched for the G1 phase, indicating a stronger effect on mRNA and protein synthesis (Fig. 2a). Consistent with melanoma biopsies, the vast majority of human melanoma cell lines from the cancer cell line encyclopaedia [27] (CCLE) expressed medium to high HECTD2 levels, with the exception of SK-MEL-3 and IGR-1 cells, where HECTD2 levels were very low (Fig. 2b). Inspection of cancer dependency map (DepMap) data [28] indicated that HECTD2 is not an essential gene in cell lines from multiple cancer types, including melanoma (Fig. S1a). Although the loss of HECTD2 function was variable and overall neutral for cell lines derived from metastatic melanoma, a potentially negative effect on growth was observed for lines derived from primary melanoma, in proportion with HECTD2 expression (Fig. 1a). To examine a direct effect on cell growth, we stably overexpressed HECTD2 in IGR-1 cells by retroviral transduction (Fig. S1b). Compared with parental cells, IGR-1 cells overexpressing HECTD2 (IGR-1.HECTD2) exhibited decreased cell and dry mass duplication times and considerably reduced cell perimeter and area (Fig. 2c, b), consistent with accelerated proliferation, which was further confirmed by analysis of total cell counts and total dry mass accumulated over time (Fig. 2e), and with live imaging of cell growth (Video S1).

To extend these findings, we examined the potential role of HECTD2 in murine melanoma. The murine and human HECTD2 proteins share $89.3 \%$ amino acid identity, indicating a highly conserved function in the two species (Fig. S2a). Moreover, Hectd2 was highly expressed in commonly used murine melanoma cells lines, with the exception of $\mathrm{HCmel} 31$ cells, where it was expressed at much lower levels, determined by RT-qPCR (Fig. 3a). Similar to the human HECTD2 locus, the murine Hectd2 locus demonstrates both sense and antisense transcription, initiated at syntenic positions (Fig. S2b). However, in contrast to the human antisense transcript, which terminates at the human-specific $H E R V H$ integration, the murine antisense transcript, Hectd2os does not span the Hectd2-encoding locus and, importantly, is not anticorrelated with sense transcription (Fig. S2b, c). Thus, although the regulation of its transcription differs between mouse and man, HECTD2 expression characterises both human and murine melanoma cell lines.

To examine a direct effect on cell growth, we stably overexpressed murine HECTD2 in $\mathrm{HCmel} 31$ cells by retroviral transduction (Fig. S3a-c), together with a GFP reporter separated by an internal ribosome entry site. Two clones were selected, expressing slightly different HECTD2 levels (Fig. S3a-c). As a control, we also overexpressed a C742A mutant of murine HECTD2 with a predicted loss of the catalytic activity (Fig. S3b; Fig. S4; Supplementary text).

In keeping with its effects on human IGR-1 cells, overexpression of HECTD2 in murine $\mathrm{HCmel} 31$ cells had a dramatic effect on cell size and granularity, as determined by flow cytometry, decreased the cell and dry mass duplication times and significantly reduced cell perimeter and area, as determined by microscopy (Fig. 3b-d), and accelerated cell growth, as determined by live imaging (Video S2). The observed effects were stronger in $\mathrm{HCmel} 31$. Hectd clone 1, expressing higher HECTD2 levels, but were not seen in cells expressing the C742A HECTD2 variant ( $\mathrm{HCmel31.Hectd}{ }^{\mathrm{C7} 42 \mathrm{~A}}$ ), regardless of expression levels (Figs. 3b-d; S3; Video S2).

To further quantify the proliferative advantage conferred by HECTD2 expression, we competed for parental HCmel31 cells and HECTD2-overexpressing HCmel31. Hectd cells, which were distinguished by GFP expression in the latter (Fig. S5a). The ratio of $\mathrm{HCmel31.Hectd} \mathrm{cells} \mathrm{to} \mathrm{HCmel} 31$ cells changed over 15-fold during a 3-day co-culture of cells plated at equal starting numbers, and a comparable shift was seen for co-cultures started at different ratios (Fig. S5a). In contrast, the ratio of $\mathrm{HCmel31}$. Hectd ${ }^{C 742 A}$ cells to $\mathrm{HCmel31}$ cells remained constant in their respective co-cultures for at least 7 days (Fig. S5a).

We used the same competition assay to examine the effect of HECTD2 overexpression in murine melanoma cells lines B16 and Braf $^{\mathrm{V} 600 \mathrm{E}}$, which were already spontaneously expressing high 

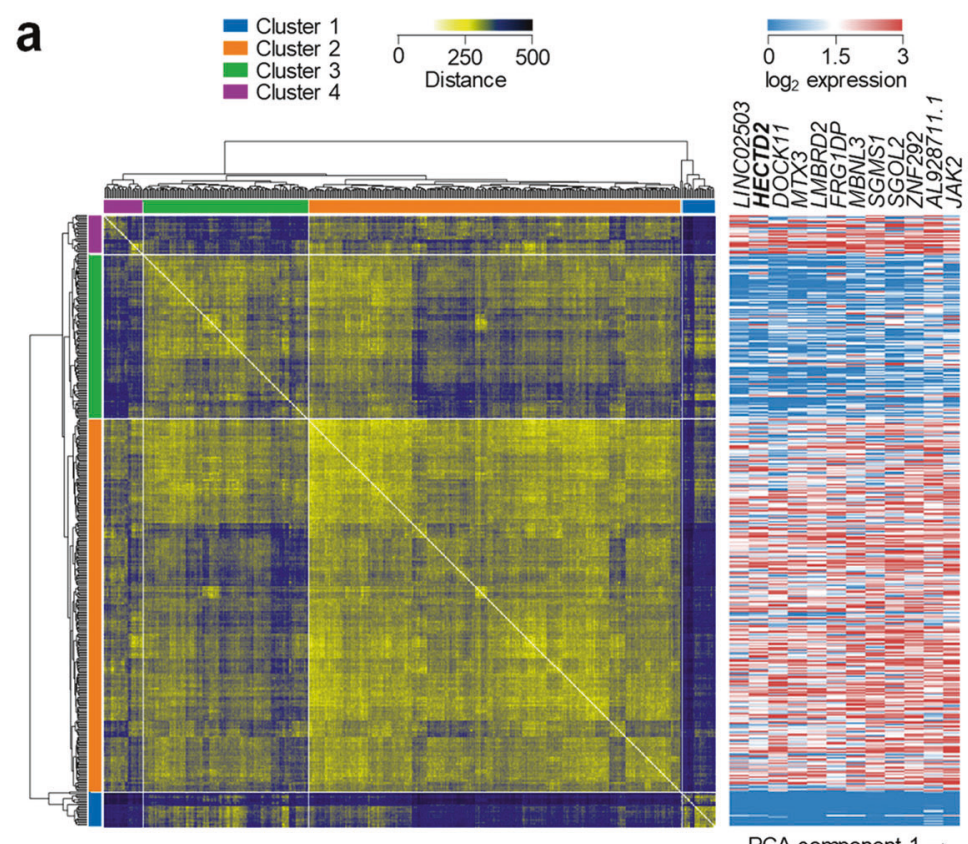

PCA component $1 \rightarrow$ b
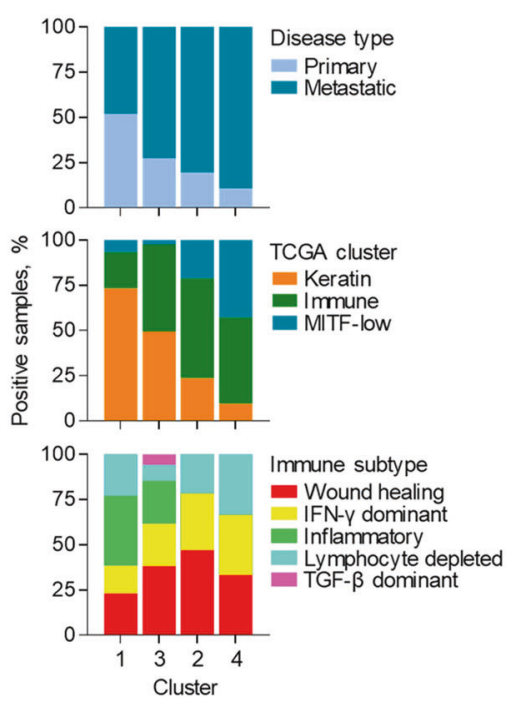

UVM

C

SKCM primary

SKCM metastatic
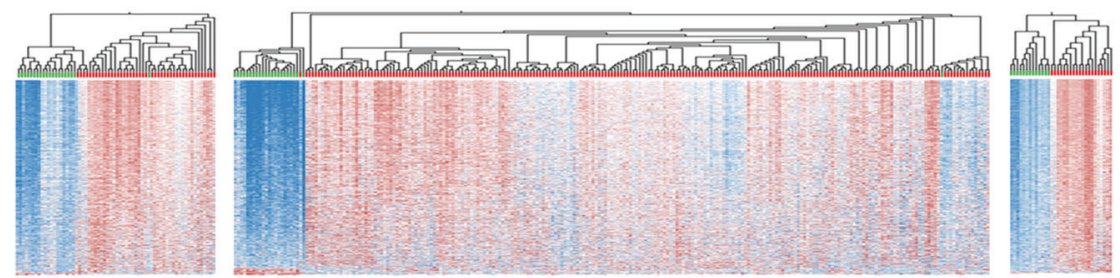

HECTD2 low
HECTD2 high

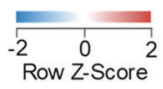

d

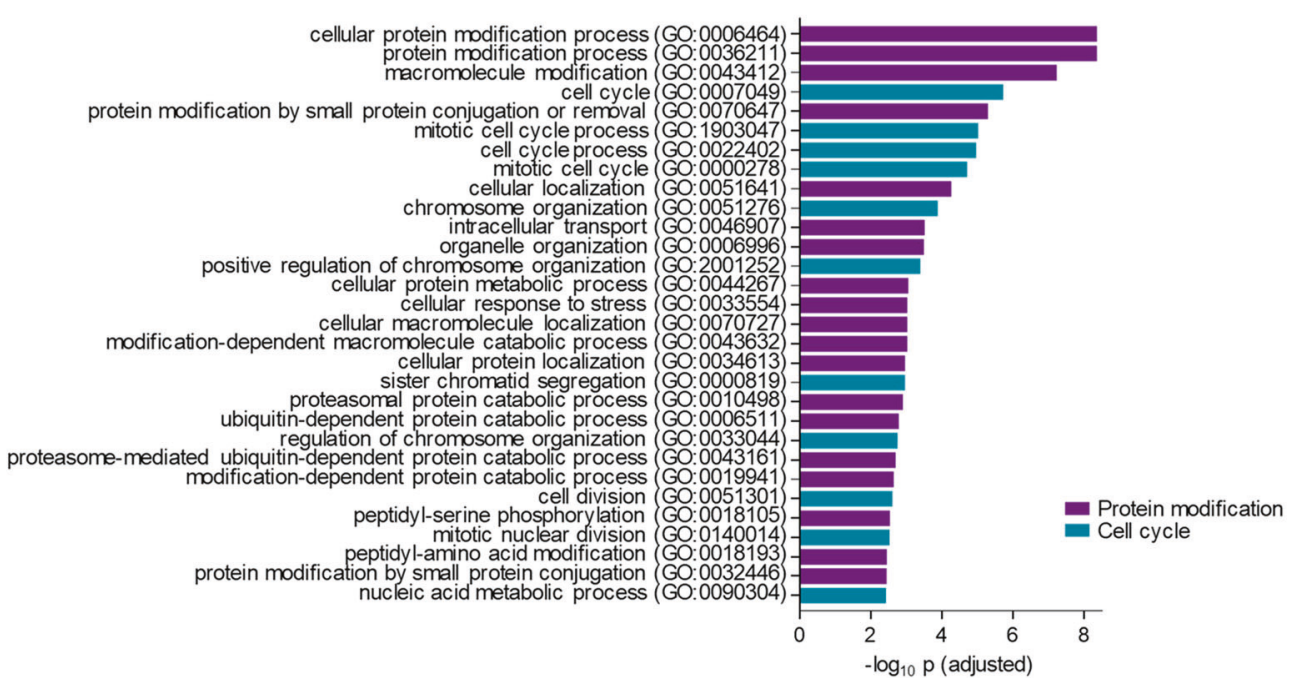

Fig. 1 HECTD2 expression stratifies melanoma patients. a Hierarchical clustering of 442 TCGA SKCM samples according to sample distance, calculated based on all gene expression (left). Expression of the top 12 genes, ranked by their contribution to sample distance (right). Each row represents the same sample in both heatmaps. b Correspondence between each individual cluster defined by sample distance and previously described molecular clusters. c Hierarchical clustering of primary and metastatic SKCM samples and UVM samples, according to differential gene expression between low and high HECTD2 expressing subsets ( $\geq 2$-fold, $q \leq 0.05)$. d Gene functional annotation analysis of the top 1000 genes upregulated in high HECTD2 expressing samples.

levels of HECTD2. Further increasing HECTD2 expression B16 and Braf $^{\mathrm{V} 600 \mathrm{E}}$ cells had no measurable effect on their growth, when competed with the respective parental cell lines (Fig. S5c, d), likely because HECTD2 expression in these cells was already mediating the maximal effect. We, therefore, performed the reverse experiment of loss of HECTD2 function in Braf ${ }^{\mathrm{V} 600 \mathrm{E}}$ cells. To this end, we used Cas9-mediated disruption of the Hectd2 gene in these cells by introducing a promoterless GFP-encoding open reading frame into Hectd2 exon 1 (Fig. S6). Expression of GFP in these cells reports insertion in the correct position with respect to 

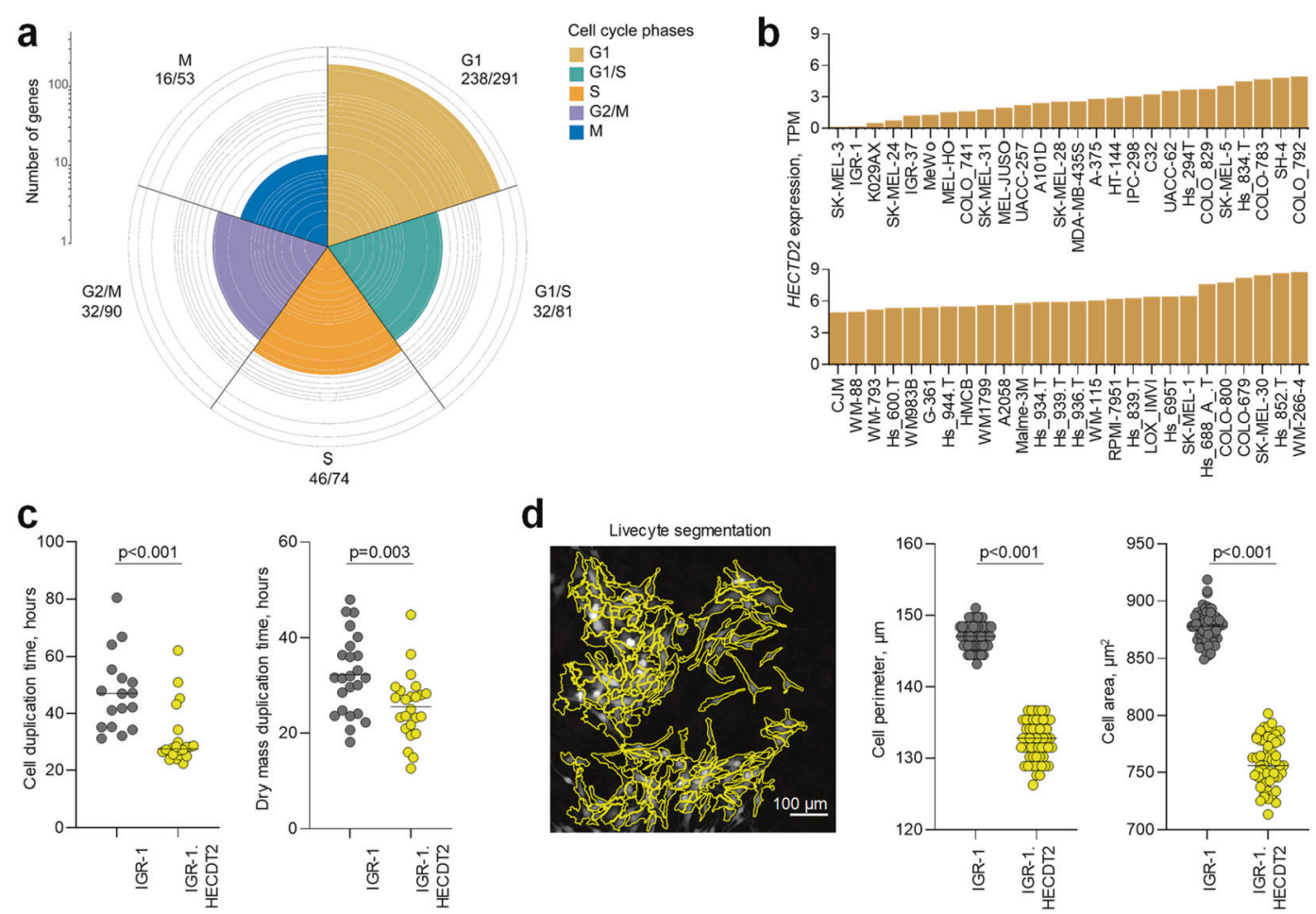

d
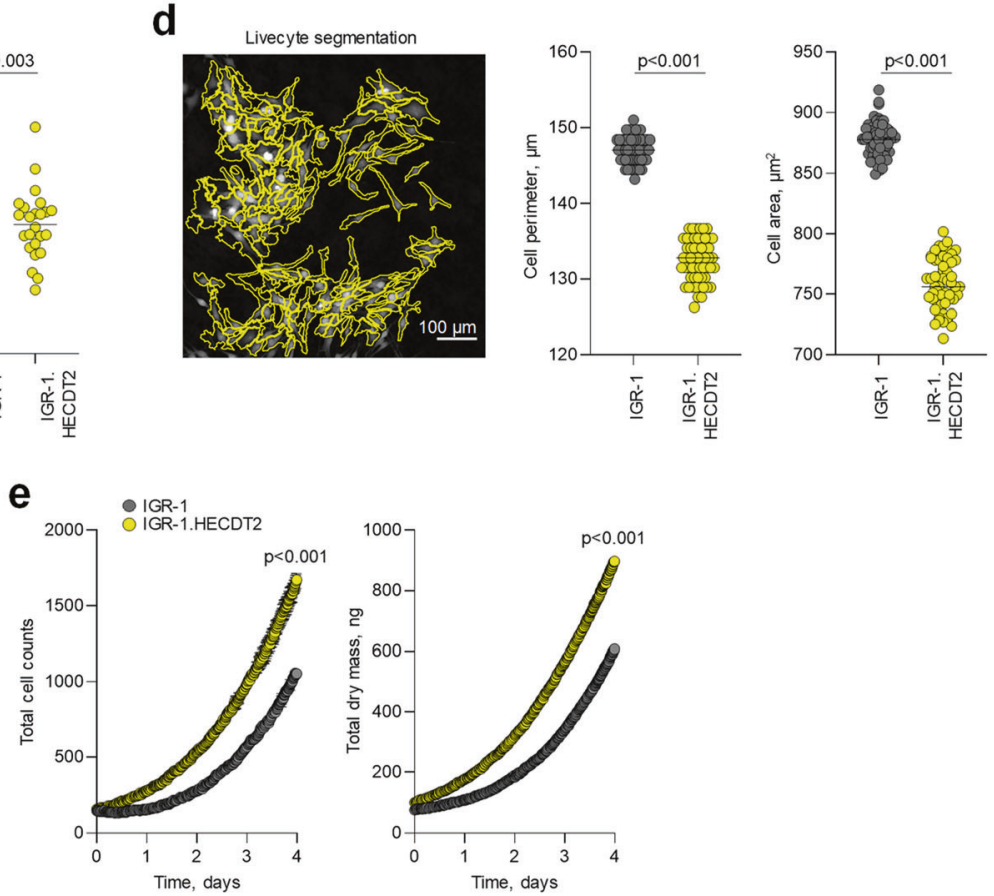

Fig. 2 HECTD2 expression drives the cell cycle in human melanoma cell lines. a Distribution of genes differentially upregulated in high HECTD2 expressing samples, according to the phase of the cell cycle they are functionally annotated to be involved in. $\mathbf{b}$ Expression of $H E C T D 2$ (TPM) in the indicated human melanoma cells lines in data from CCLE. c Cell duplication times (left) and dry mass duplication times (right) of parental IGR-1 human melanoma cells and IGR-1 cells overexpressing HECTD2 (IGR-1.HECTD2). Symbols represent the mean values of separate wells $(n=24)$, with 4 fields of view averaged per well, from three independent experiments. $\mathbf{d}$ Representative example of cell perimeter and cell area demarcation using Livecyte segmentation (left) and cell perimeters and cell areas of IGR-1 and IGR-1.HECTD2 cells. Symbols represent individual cells in one of three independent experiments. e Total cell counts (left) and total dry mass (right) in cultures of IGR-1 and IGR-1. HECTD2 cells over time. Plotted are the mean values $( \pm$ SEM) of the mean of each of the three independent experiments. $P$ values were calculated with Mann-Whitney Rank Sum tests.

the locus promoter (Fig. S6). Targeted cells were sorted on the basis of GFP expression to over $90 \%$ purity, but their frequency was reduced to under $60 \%$ after a two-week culture (Fig. S6b). $\mathrm{GFP}^{+}$cells were sorted again to a higher purity (99\%), but again were outcompeted by the residual GFP ${ }^{-}$cells following culture (Fig. S6b), and a stable $\mathrm{GFP}^{+}$subline could not be established. Thus, high spontaneous expression of HECTD2 in Braf ${ }^{\mathrm{V} 600 \mathrm{E}}$ cells appeared necessary for maximal cell growth in vitro.

To independently confirm these results, we attempted to block HECTD2 activity using the recently developed BC-1382 small molecule inhibitor [22]. In cultures of $\mathrm{Braf}^{\mathrm{V} 600 \mathrm{E}}$ and $\mathrm{HCmel} 31$ cells, the addition of BC-1382 significantly increased cell and dry mass duplication times, as well as cell perimeter over time (Fig. 4). These changes were equivalent to a 4.8 -fold and 2.0 -fold reduction in cell accumulation over 5 days of culture, for Braf ${ }^{\mathrm{V} 600 \mathrm{E}}$ and $\mathrm{HCmel} 31$ cells, respectively. The magnitude of this effect, at least for $\mathrm{HCmel} 31$ cells, was not different from the non-specific effect of
BC-1382 on HECTD2-negative U937 cells (1.9-fold reduction; Fig. S7; Supplementary text) and could not, therefore, be attributed to HECTD2 inhibition. In contrast, the addition of BC-1382 to HCmel31. Hectd $c 1$ cells had a much stronger effect on the same parameters, equating to a 16.3-fold reduction in cell accumulation in the same time period, fully reversing the effects of HECTD2 overexpression in these cells (Fig. 4). Collectively, these results support a major role for catalytically-active HECTD2 in driving the cell-autonomous proliferation of human and murine melanoma cell lines.

\section{HECTD2 tunes immune-reactivity to melanoma growth}

Although HECTD2 clearly promoted in vitro growth of melanoma cell lines, in vivo tumour growth is influenced by additional tumour-extrinsic factors, including the anti-tumour immune response. A pro-inflammatory role for HECTD2 in the healthy lung has been proposed [22] and it was, therefore, possible that high HECTD2 expression in melanoma was also pro-inflammatory. 
a

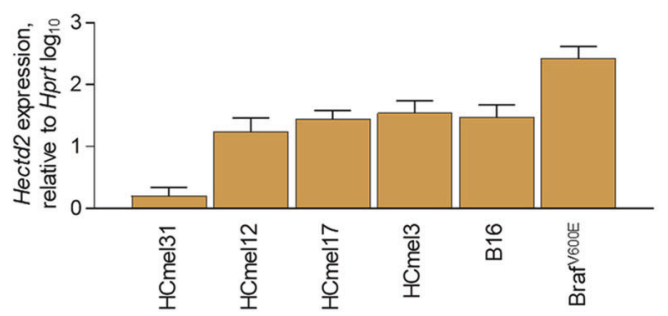

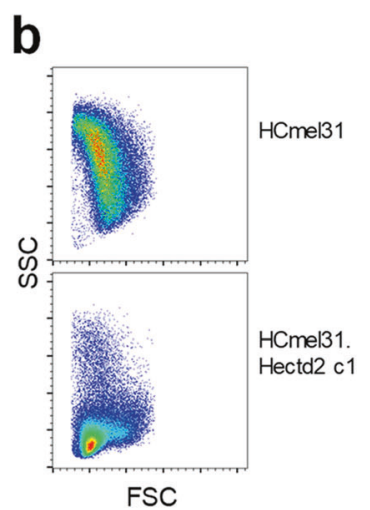

d

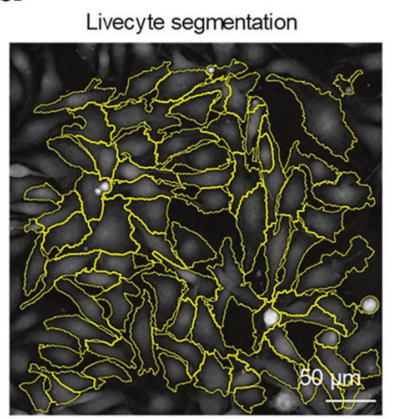

C
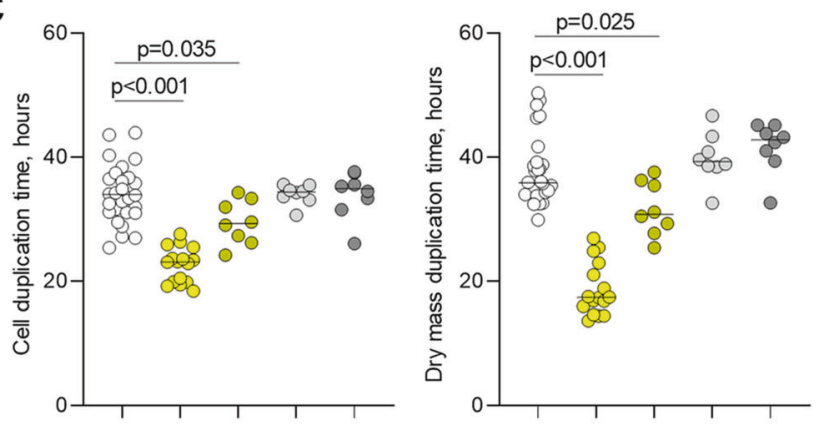

HCmel31 $\mathrm{HCmel} 31$. Hectd2 $\mathrm{c} 1 \quad \bigcirc \mathrm{HCmel31.Hectd2C742 \textrm {A }} \mathrm{c1}$

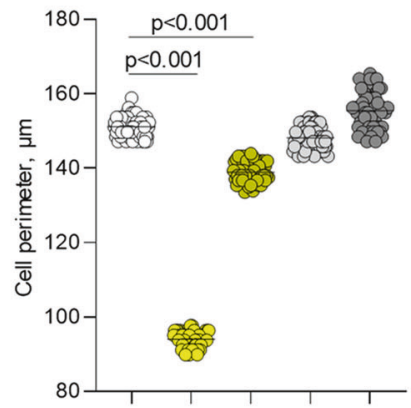

Fig. 3 HECTD2 expression drives the cell cycle in murine melanoma cell lines. a Expression of Hectd2 in the indicated murine melanoma cells lines, measured by RT-qPCR. b Flow cytometric example of forwarding and side scatters of parental HCmel 31 murine melanoma cells and $\mathrm{HCmel} 31$ clone 1 cell overexpressing Hectd2 (HCmel31.Hectd2 c1). c Cell duplication times (left) and dry mass duplication times (right) of parental HCmel31 and HCmel31 cells overexpressing wt or C742A HECTD2 variant proteins (two clones for each). Symbols represent the mean values of separate wells $(n=8-28)$, with four fields of view averaged per well, from 1 to 4 independent experiments. $\mathbf{d}$ Representative example of cell perimeter and cell area demarcation using Livecyte segmentation (left) and cell perimeters and cell areas of the same HCmel31 cell line derivatives. Symbols represent individual cells in one of three independent experiments. $P$ values were calculated with One Way Analysis of Variance (ANOVA) on Ranks tests.

To examine the overall effect of HECTD2 expression on melanoma growth in vivo, we employed the three transplantable murine cell lines, $\mathrm{HCmel} 31, \mathrm{Braf}^{\mathrm{V} 600 \mathrm{E}}$ and $\mathrm{B} 16$, and monitored tumour formation and infiltration by diverse immune cell types (Fig. S8). $\mathrm{HCmel} 31$ cells transplanted into fully-syngeneic C57BL6/J (B6) mice grew into tumours over a period of 30-50 days, without eliciting an overt immune reaction (Fig. 5a, b), as previously reported [29, 30]. In stark contrast, the growth of $\mathrm{HCmel} 31$. Hectd cells transplanted into such recipients was significantly accelerated (7-14 days) and was also accompanied by immune infiltration of the tumours (Fig. 5a, b). Immune infiltrates comprised diverse immune cell types, dominated by myeloid cells, but contained relatively few antigen-experienced (CD44 ${ }^{\text {high }}$ ) $T$ cells (Figs. 5b and S8). Accordingly, despite the recruitment of immune cells in the $\mathrm{HCmel} 31$. Hectd tumours, their growth was unaffected by an adaptive immune response, as comparably accelerated growth of $\mathrm{HCmel31.Hectd} \mathrm{cells} \mathrm{were}$ observed also in severely immunodeficient $\mathrm{Rag}^{-1-} \mid 12 \mathrm{rg}^{-1-} \mathrm{Cd} 47^{-1-}$ recipients, lacking all lymphocytes (Fig. S9), demonstrating a tumour cell-intrinsic effect of HECTD2 expression. Therefore, overexpression of HECTD2 in HCmel31 cells autonomously increased their in vivo growth and ignited immune infiltration of tumours formed, which, however, did not restrain their growth.

As previously reported [29-33], Braf ${ }^{\mathrm{V} 600 \mathrm{E}}$ cells injected into B6 mice formed tumours with faster kinetics than $\mathrm{HCmel} 31$ cells (Fig. 5c). Moreover, Braf ${ }^{V 600 E}$ tumours were heavily infiltrated by immune cells, predominantly myeloid cells, often outnumbering tumour cells, with very few antigen-experienced CD44 high $T$ cells or dendritic cells (Fig. 5c). HECTD2 overexpression in Braf ${ }^{\mathrm{V} 600 \mathrm{E}}$ cells had no measurable effect on either growth or immune infiltration (Fig. 5c, d). Similarly, injection of B16 cells into B6 mice led to rapid tumour growth and immune infiltration by low overall numbers of immune cells, but containing a higher proportion of antigenexperienced CD44 ${ }^{\text {high }}$ T cells and dendritic cells (Fig. 5e, f). HECTD2 overexpression in B16 cells caused a slight delay (1-2 days) in tumour growth but did not appreciably alter the outcome of immune response to the rumours (Fig. 5e, f). Thus, consistent with 
untreated

vehicle

BC-1382
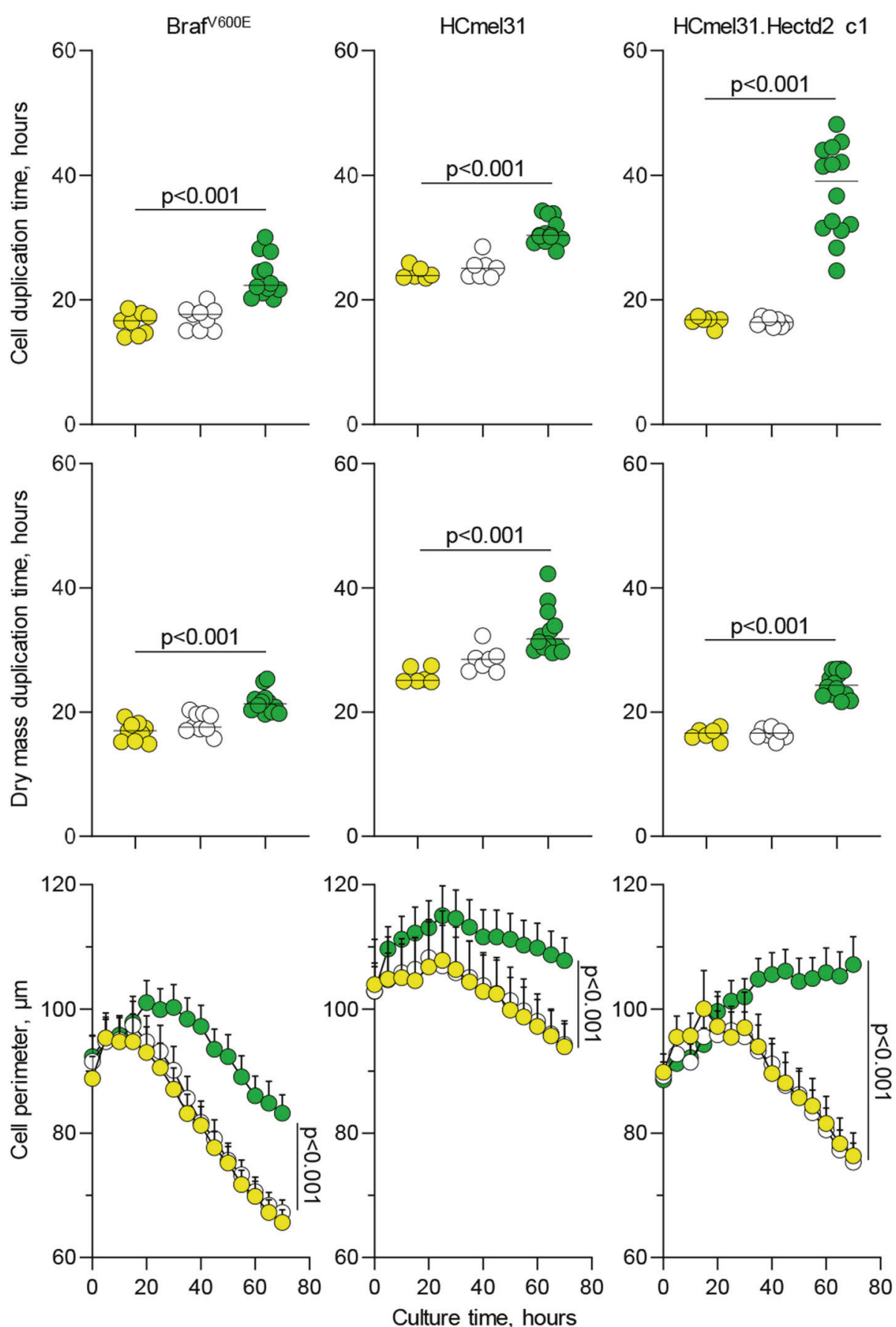

Fig. 4 Deceleration of cell proliferation by in vitro HECTD2 inhibition. In vitro growth of parental Braf ${ }^{\mathrm{V} 600 \mathrm{E}}$ cells, $\mathrm{HCmel} 31 \mathrm{cells}$ and $\mathrm{Hectd} 2-$ overexpressing HCmel31. Hectd2 c1 cells, in the absence of treatment or in the presence of the $200 \mu \mathrm{M} \mathrm{BC}-1382$ inhibitor or of the DMSO (vehicle) alone. Mean cell duplication times (top) and dry mass duplication times (middle) of separate wells $(n=9-12)$, with four fields of view averaged per well, are shown from 3 to 4 independent experiments. $P$ values were calculated with ANOVA on Ranks tests. Mean cell perimeters $( \pm$ SEM) from separate wells $(n=9-12)$ are shown (bottom) from three independent experiments. $P$ values were calculated Student's $t$-tests.

in vitro data, in vivo growth of $\mathrm{HCmel31}$, Braf ${ }^{\mathrm{V} 600 \mathrm{E}}$ and B16 cells correlated with their spontaneous HECTD2 expression and was dramatically increased by HECTD2 overexpression specifically in HCmel31 where HECTD2 was not already highly expressed. Similarly, HECTD2 overexpression increased the immune infiltration of otherwise immune-depleted $\mathrm{HCmel} 31$ tumours but did not further enhance the infiltration of more immunogenic Braf ${ }^{\mathrm{V} 600 \mathrm{E}}$ and B16 tumours.

The combination of these findings suggested that a potential anti-tumour immune response promoted by the pro-inflammatory 


\section{a $-0-\mathrm{HCmel} 31$}

- HCmel31. Hectd2 c1

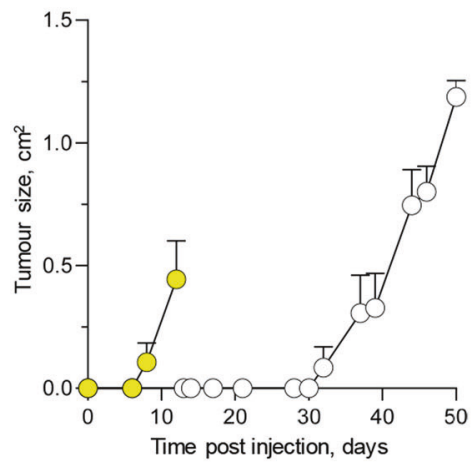

C -O-Braf $1600 E$ - Braf ${ }^{\mathrm{V} 600 \mathrm{E}}$.Hectd2

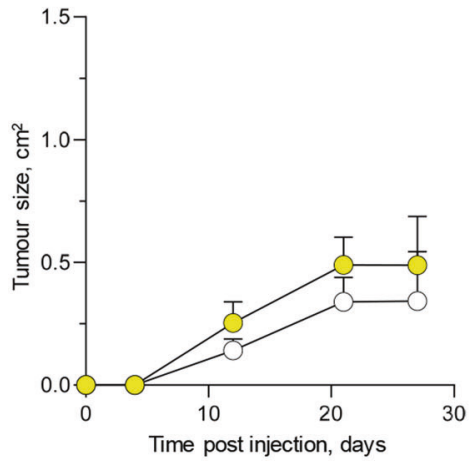

e $\quad$ B16 - B16.Hectd2

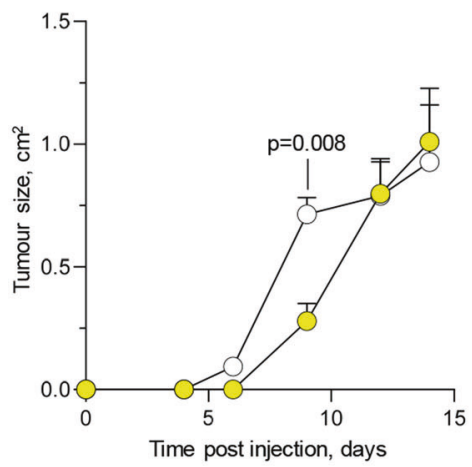

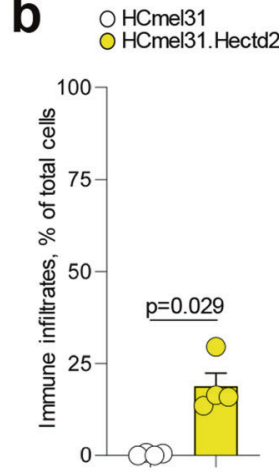

d
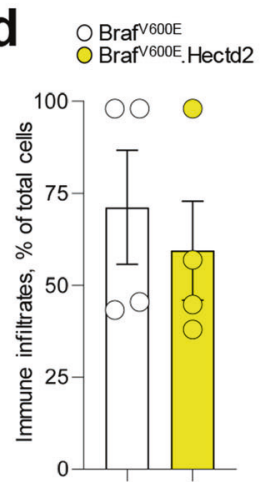

f

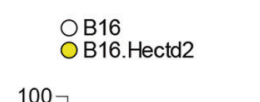

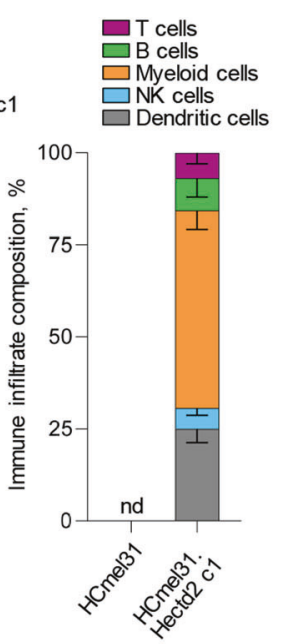

O HCmel31
O HCmel31. Hectd2 c1
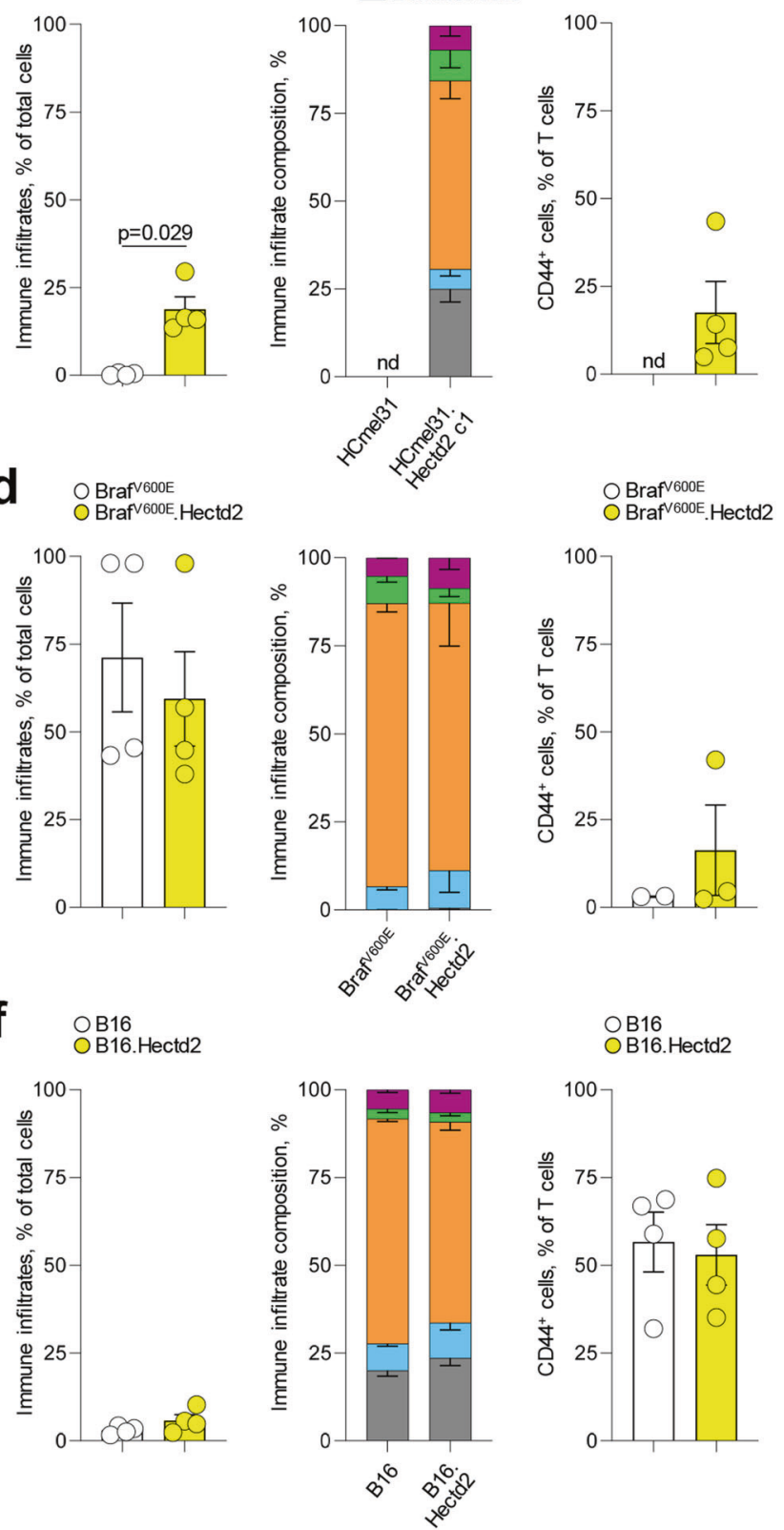

Fig. 5 Effect of HECTD2 overexpression on murine melanoma growth in vivo. a Mean size ( \pm SEM) of tumours formed over time following injection of $\mathrm{HCmel} 31$ cells or HCmel31.Hectd2 $\mathrm{c} 1$ cells into B6 recipients ( $n=4$ and $n=4$, respectively, from one of two experiments). b Percentage and composition of $\mathrm{CD}_{4} 5^{+}$immune infiltrates, and percentage of antigen-experienced CD44 ${ }^{+}$cells in tumour-infiltrating T cells in the same recipients as in a. Bars graphs denote the mean ( \pm SEM) and symbols represent individual mice. c Mean size ( \pm SEM) of tumours formed over time following injection of Braf ${ }^{\mathrm{V} 600 \mathrm{E}}$ cells or Braf ${ }^{\mathrm{V} 600 \mathrm{E}}$. Hectd2 cells into B6 recipients $(n=8$ and $n=8$, respectively, from two experiments). d Percentage and composition of $\mathrm{CD}^{+} 5^{+}$immune infiltrate, and percentage of antigen-experienced CD44 ${ }^{+}$cells in tumourinfiltrating $T$ cells in the same recipients as in c. Bars graphs denote the mean ( \pm SEM) and symbols represent individual mice. e Mean size $( \pm$ SEM) of tumours formed over time following injection of B16 cells or B16. Hectd2 cells into B6 recipients $(n=4$ and $n=4$, respectively, from one of two experiments). f Percentage and composition of $\mathrm{CD}_{4} 5^{+}$immune infiltrates, and percentage of antigen-experienced CD44 ${ }^{+}$cells in tumour-infiltrating $T$ cells in the same recipients as in e. Bars graphs denote the mean $( \pm S E M)$ and symbols represent individual mice.

activity of HECTD2 was either non-functional or counteracted by a stronger immune-suppressive activity. To explore these possibilities, we measured the transcription levels of immune mediators that could be cell-intrinsically correlated with HECTD2 activity. Given the recently described role for HECTD2 in potentiating NF-KB activity in response to innate immune stimuli [22], we included in our analysis NF-KB target genes and also stimulation with lipopolysaccharide
(LPS), lysophosphatidic acid (LPA) and IFN- $\gamma$, which activate NF-kB. Overexpression of HECTD2 in HCmel31 cells caused a marked increase (over one million-fold) in the transcription of Ptgs2, the gene encoding cyclooxygenase 2 (COX2), also known as prostaglandin G/H synthase 2 (PGHS2), independently of innate immune stimulation (Fig. 6a). Additionally, HECTD2 overexpression in $\mathrm{HCmel31}$ cells significantly increased transcription levels of $\mathrm{CCl}$, 
a
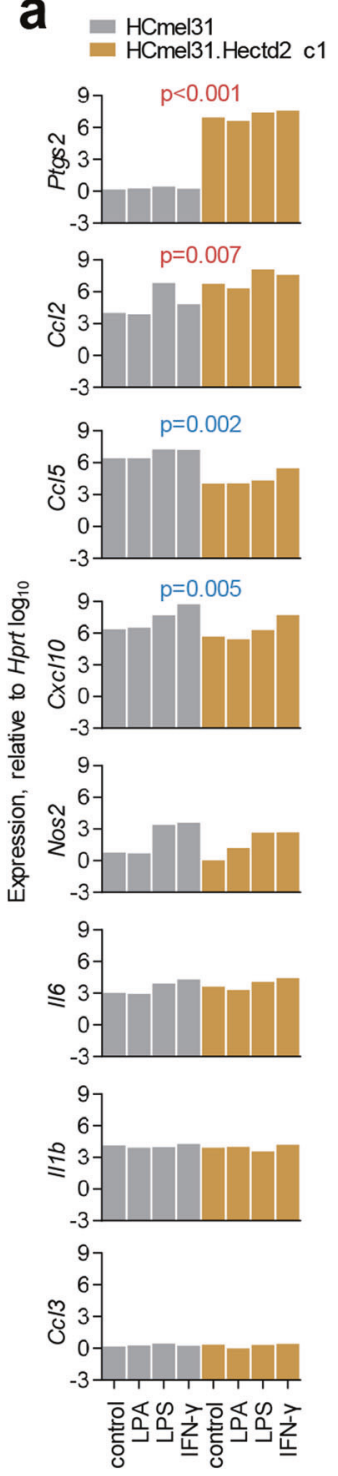

d

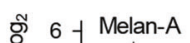

סㅇ 6 - Melan-A

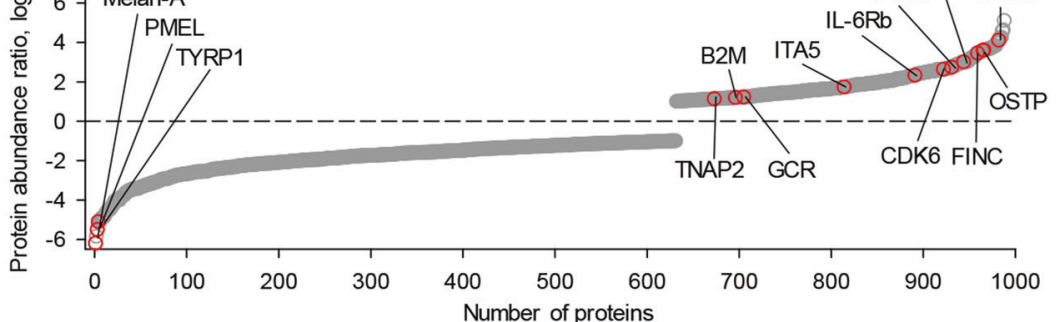

b
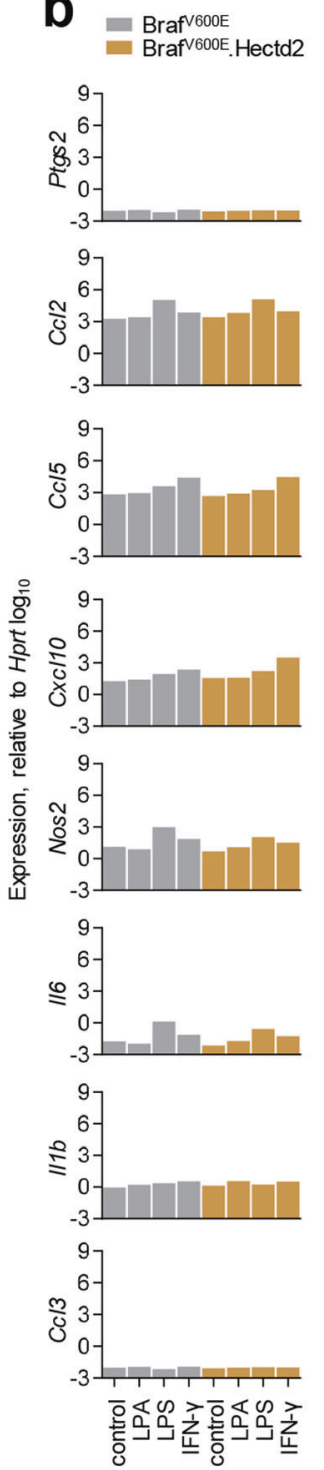

C ${ }^{B 16}$

B16. Hectd2
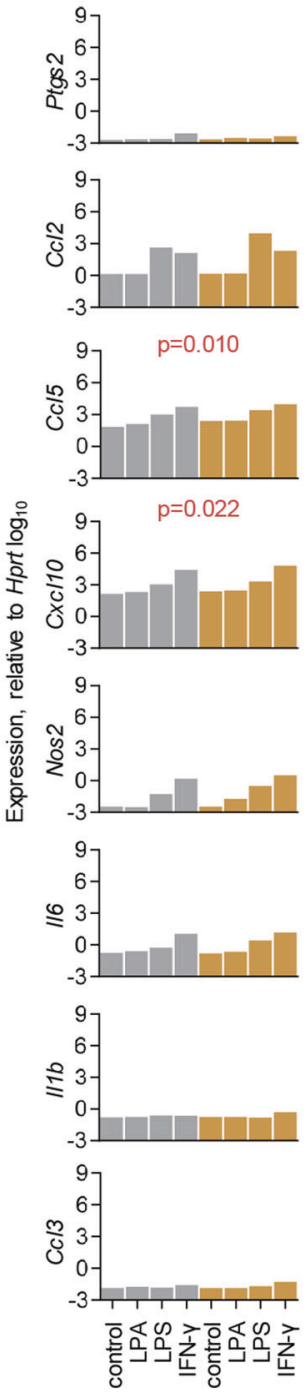

Fig. 6 Effect of HECTD2 overexpression on in vitro melanoma cell expression of NF-kB target genes encoding pro- and anti-inflammatory mediators. a-c, Production of the indicated mediators, assessed by RT-qPCR in parental HCmel31 (a), Braf ${ }^{\mathrm{V} 600 \mathrm{E}}$ (b) and B16 cells (c) and the respective Hectd2-overexpressing derivatives, either spontaneously or following stimulation with LPA, LPS or IFN- $\gamma$. Bars denote the means of technical RT-qPCR triplicates. $P$ values are calculated by paired comparisons between parental and Hectd2-overexpressing sublines for each treatment condition (Student's paired $t$-test). $\mathrm{P}$ values in red and blue indicate significant upregulation and downregulation, respectively, in Hectd2-overexpressing sublines. d Ratio of protein abundance between $\mathrm{HCmel} 31$. Hectd $2 \mathrm{c} 1$ and parental $\mathrm{HCmel} 31 \mathrm{cells}$ for the 990 individual proteins that were found to be differentially abundant ( $\geq 2$-fold, $p \leq 0.05, q \leq 0.05$ ) between the two cell lines, ranked according to their ratio.

encoding $\mathrm{CCL} 2$, also known as monocyte chemoattractant protein1 (MCP-1) (Fig. 6a). CCl2 transcription was responsive to LPS and IFN$\gamma$ stimulation, but the enhancing effect of HECTD2 overexpression was evident both in stimulated and unstimulated cells (Fig. 6a).
Transcription levels of $\mathrm{C} C \mathrm{I} 5$ and $\mathrm{CxCl} 10$ were also responsive to LPS and IFN- $\gamma$ stimulation in $\mathrm{HCmel} 31$ cells, but, in contrast to levels of $\mathrm{CCl} 2$, they were significantly reduced by HECTD2 overexpression both in stimulated and unstimulated cells (Fig. 6a). Nos2 and $1 / 6$ 
a

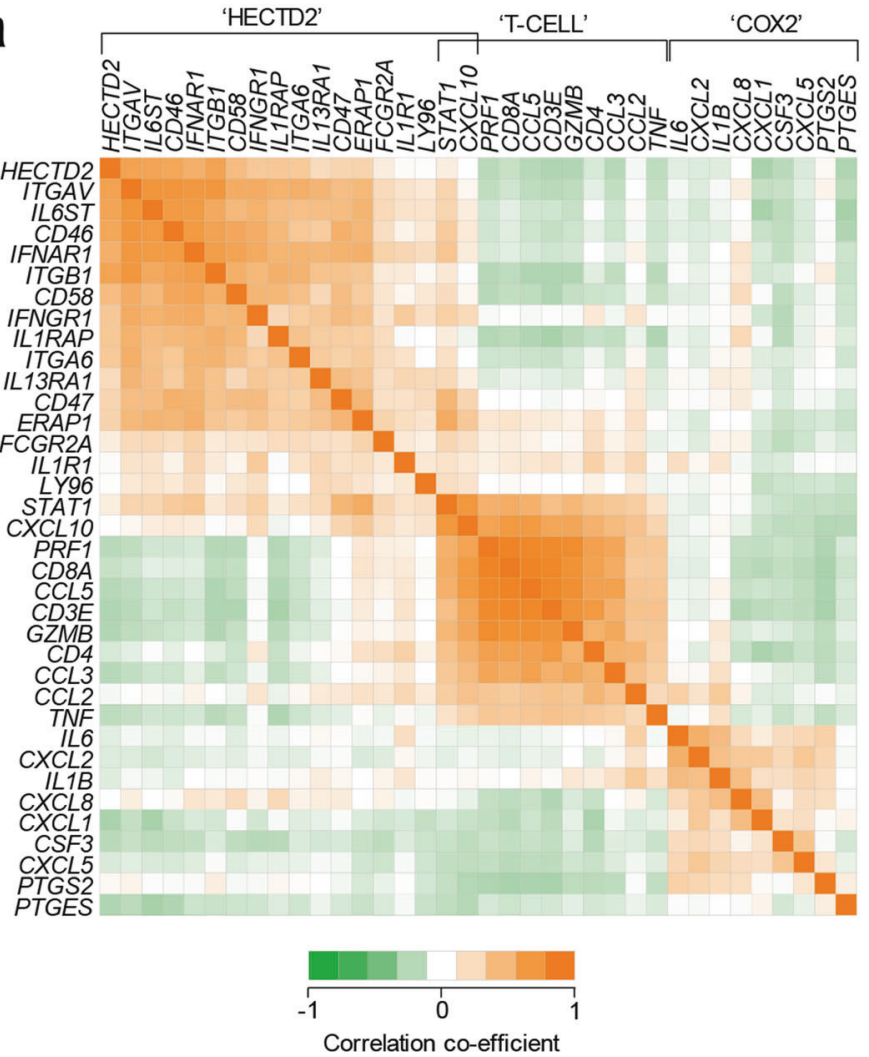

b
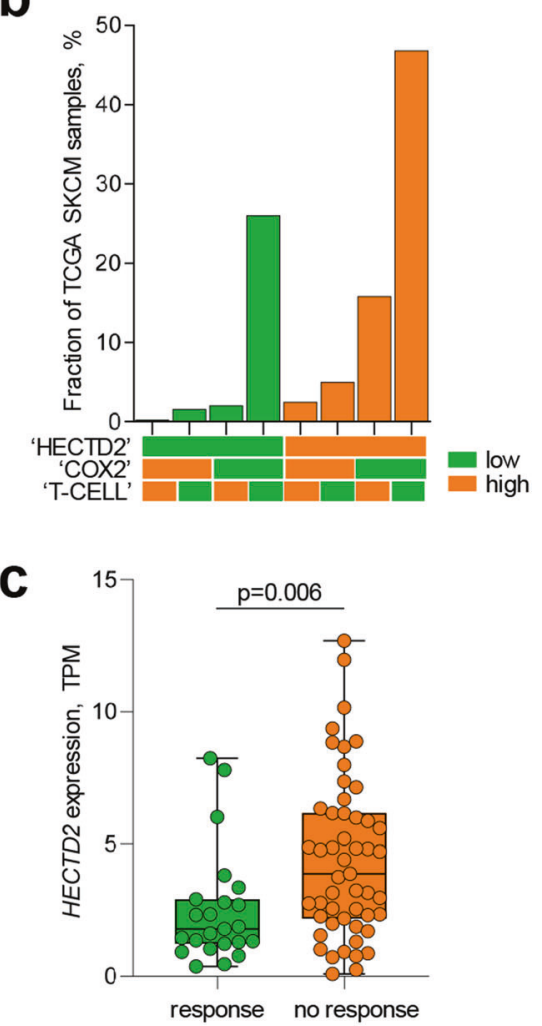

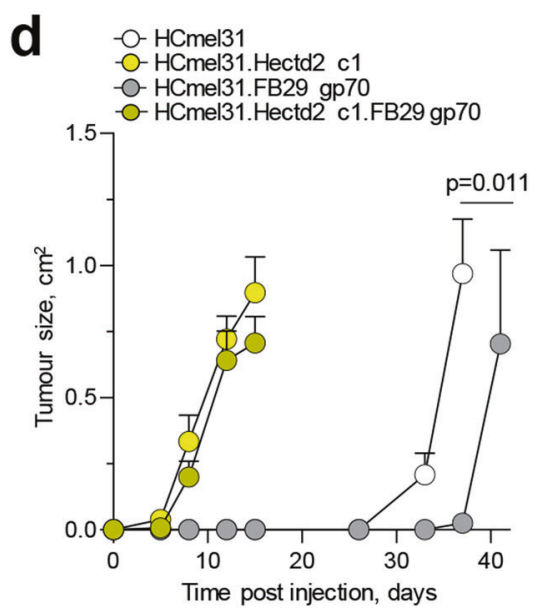

e

O HCmel31. Hectd2 c1 HCmel31.Hectd2 c1.FB29 gp70
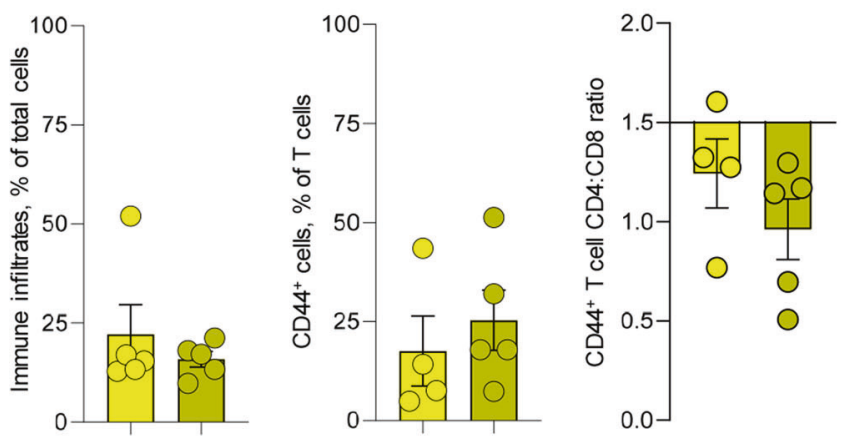

Fig. 7 Effect of HECTD2 expression on human melanoma immunotherapy and murine melanoma antitumour immunity. a Correlation matrix of expression levels of genes associated with the 'HECTD2', 'T-CELL' and 'COX2' modules in 442 TCGA SKCM samples. b Percentage of 442 TCGA SKCM samples that express the indicated combination of the 'HECTD2', 'T-CELL' and 'COX2' gene modules. c HECTD2 expression (TPM) in melanoma biopsies from patients exhibiting a response $(n=23)$ or no response $(n=51)$ to anti-PD- 1 immunotherapy $(p$ value calculated with Mann-Whitney Rank Sum test). Box plots show the upper and lower quartiles, centre lines show the median and whiskers represent the $1.5 \mathrm{x}$ interquartile range. Symbols show all individual samples. $\mathbf{d}$ Mean size $( \pm \mathrm{SEM})$ of tumours formed over time following injection into B6 recipients of $\mathrm{HCmel} 31$ cells or $\mathrm{HCmel} 31$.Hectd $2 \mathrm{c} 1$ cells and derivatives of these engineered to express FB29 gp70, $(n=4, n=$ $7, n=3$ and $n=7$, respectively). e Percentage of CD45 ${ }^{+}$immune infiltrates, percentage of antigen-experienced CD44 ${ }^{+}$cells in tumourinfiltrating T cells and CD4:CD8 ratio in antigen-experienced tumour-infiltrating T cells in the same recipients as in d. Bars graphs denote the mean $( \pm S E M)$ and symbols represent individual mice. The CD4:CD8 ratio was significantly lower $(p=0.002)$, Student's $t$-test in antigenexperienced T cells infiltrating $\mathrm{HCmel} 31$.Hectd2 c1.FB29 gp70 tumours than in naïve B6 recipients.

transcription responded to LPS and IFN- $\gamma$ stimulation, but remained unaffected by HECTD2 overexpression, whereas $/ 11 b$ and $\mathrm{CCl} 3$ transcription was minimally affected by innate immune stimulation or HECTD2 overexpression in HCmel31 cells (Fig. 6a). In agreement with the differential effects on growth or in vivo immune infiltration of the three murine cell lines, a further expression of HECTD2 did not affect transcription of these immune mediators in Braf ${ }^{\mathrm{V} 600 \mathrm{E}}$ cells and only moderately increase $\mathrm{C} C / 5$ and $\mathrm{Cxcl} 10$ transcription in B16 cells (Fig. $6 \mathrm{~b}, \mathrm{c}$ ), again illustrating the different melanoma profiles exemplified by these three cell lines.

To independently, as well as more comprehensively, validate the observed effects of HECTD2 overexpression on NF-KB target genes, we compared the global proteomic profiles of parental and HECTD2-overexpressing HCmel31 cells. HECTD2 overexpression 
leads to loss of melanosome-specific proteins Melan-A, PMEL and TYRP1, and to the significant overrepresentation of several known NF-KB targets, including COX2 (also known as PGHS2, encoded by the Ptgs2 gene) and its homologue COX1 (also known as PGHS1, encoded by the Ptgs1 gene), upregulated 6.7-fold and 17.2-fold, respectively (Fig. 6d; Table S1; Fig. S10; Supplementary text). Collectively, these data confirm the direct link between HECTD2 overexpression and increased NF-KB activity, which likely underlies the effect of HECTD2 on the cell cycle and immunogenicity of tumour cells.

\section{HECTD2 counteracts adaptive immune resistance to melanoma}

The results from the murine $\mathrm{HCmel} 31$ cells demonstrated that HECTD2 overexpression modified the balance of the proinflammatory and immune-suppressive mediators we analysed. To examine if this effect on the selected immune mediators reflected broader changes in immune signatures and also in human melanoma, we build modules of co-regulated immune genes.

In TCGA SKCM, expression of PTGS2 was highly correlated with expression of PTGES, encoding prostaglandin $E$ synthase, in a module we refer to as 'COX2' to denote the COX2-mediated prostaglandin $E_{2} \quad\left(P_{E_{2}}\right)$ biosynthetic pathway (Fig. 7a). In agreement with prior reports [33], this module was characterised by higher expression of $I L 6, I L 1 B$ and CSF3, as well as of several neutrophil chemoattractants (Fig. 7a). Also agreeing with prior reports [33], the 'COX2' module was distinct from the 'T-CELL' module, characterised by expression of genes from $\mathrm{CD}^{+}$and cytotoxic $\mathrm{CD}^{+}{ }^{+}$cells and chemokine genes CCL2, CCL3, CCL5 and CXCL10 (Fig. 7a).

Despite the stronger association with protein modification and cell cycle pathways (Fig. 1d), high expression of HECTD2 in TCGA SKCM was also associated with a number of co-regulated immune genes (Fig. 7a). These formed a separate module, referred to as 'HECTD2', which was distinct from the 'COX2' module and showed only a small overlap (STAT1, CXCL10) with the 'T-CELL' module (Fig. 7a). The upregulated genes in the 'HECTD2' module encoded predominantly transmembrane proteins, such as integrins and adhesion molecules (ITGAV, ITGB1, ITGA6 and CD58), and receptors and co-receptors for interleukins (IL1R1, IL6ST, IL13RA1), interferons (IFNAR1 and IFNGR1), immunoglobulins (FCGR2A), complement (CD46) and LPS (LY96) (Fig. 7a). Also notable was the co-expression in the 'HECTD2' module of CD47 (Fig. 7a), which encodes CD47, also known as integrin associated protein (IAP), and which can inhibit phagocytosis in macrophages [34].

We next stratified 442 TCGA SKCM samples according to the aggregate expression of the genes in each of these three distinct modules and looked for the overlap in expression. As expected by the segregation observed at the gene level (Fig. 7a), the expression of the modules in SKCM samples also largely segregated into distinguishable subsets (Fig. 7b). The 'COX2' module was expressed in a minority of SKCM samples (41 of 442, 9.3\%), most of which were within the 'HECTD2'-expressing subset (Fig. 7b). Samples expressing the 'T-CELL' module were more numerous (91 or 442, 20.6\%), largely distinct from 'COX2'expressing samples, and most were also within the 'HECTD2'expressing subset (Fig. 7b). However, the largest single subset, amounting to nearly half of all samples (207 of 442, 46.8\%), expressed the 'HECTD2' module in the absence of the other two modules (Fig. 7b).

The partial overlap of the 'HECTD2' and 'T-CELL' modules at the gene expression level (Fig. 7a) and inclusion of the majority of the 'T-CELL'-expressing samples within the 'HECTD2'-expressing subset (Fig. 7a; $p<0.00001$, Fisher Exact test) is consistent with a proinflammatory role for HECTD2. However, the much larger number of 'HECTD2'-expressing samples without evidence of an antitumour $\mathrm{T}$ cell response, suggests either lack of inherent immunogenicity of these tumours or, alternatively, active HECTD2-mediated immune modulation, only a small part of which could be attributed to the immune suppressive ' $C O X^{\prime}$ module. To examine if elevated HECTD2 expression may correlate with a lack of effective T cell anti-tumour immunity, we analysed cohorts of melanoma patients that were treated with PD-1 blocking antibodies $[35,36]$. Notably, the outcome of such immunotherapy correlated significantly with levels of HECTD2 expression (Fig. 7c). Given that the response to PD-1 blockade was not strongly associated with tumour mutation load and, by extension, expected tumour neoantigenicity in either of these cohorts [35, 36], the link between elevated HECTD2 expression and lack of immunotherapy response suggest that HECTD2 activity may counteract $T$ cell anti-tumour immunity.

To test this hypothesis directly, we used the non-immunogenic $\mathrm{HCmel} 31$ tumour cells, which we engineered to express a retroviral antigen (Friend helper murine leukaemic virus isolate FB29 envelope glycoprotein gp70), previously shown to elicit adaptive anti-tumour responses even in the absence of prior immunisation [30, 37]. The expression of FB29 gp70 in $\mathrm{HCmel} 31$ cells significantly delayed their growth after inoculation into naïve B6 mice (Fig. 7d). In contrast, expression of FB29 gp70 had no discernible impact on the growth of HCmel31 cells that expressed high levels of HECTD2, despite the recruitment of sizeable immune infiltrates, particularly of $\mathrm{CD}^{+}{ }^{\mathrm{T}}$ cells, mediated by HECTD2 (Fig. 7d, e). Thus, although HECTD2 expression alone was sufficient to attract immune cells to the otherwise nonimmunogenic $\mathrm{HCmel} 31$ tumours, it did not promote $\mathrm{T}$ cellmediated tumour resistance and even negated the resistance provided by the immunogenic expression of FB29 gp70 as a tumour-specific antigen.

\section{DISCUSSION}

Our results place HECTD2 as a central regulator of a number of different functions that determine melanoma progression. This central role is supported by the stratification of melanoma subtypes based primarily on HECTD2 expression, forming transcriptional clusters of genes involved in protein modification and the cell cycle. Accordingly, HECTD2 expression directly promoted cell-autonomous proliferation of human melanoma cells in vitro and murine melanoma cells in vitro and in vivo. This increase in proliferation was also accompanied by extensive morphological changes at least in vitro, with a reduction in cell size as the most notable.

Another major effect of HECTD2 activity in melanoma cells is on their production of soluble immune mediators and transmembrane proteins involved in interaction with immune cells. HECTD2 overexpression in $\mathrm{HCmel} 31$ cells directly increased levels of Ptgs2 transcription by at least six orders of magnitude. Cancer cell-intrinsic expression of COX2, encoded by Ptgs2, has been shown to induce $\mathrm{PGE}_{2}$, which in turn subverts myeloid cell function in melanoma [33]. In contrast, loss or inhibition of COX2 activity promotes anti-tumour immunity, developing either spontaneous or in the context of checkpoint blockade [33]. Its effect on Ptgs2 transcription places HECTD2 upstream of this important 'COX2' module, which controls the $\mathrm{PGE}_{2}$ biosynthetic pathway, as well as expression of $I L 6, I L 1 B$, CSF3 and neutrophil chemoattractants. Induction of the immunosuppressive 'COX2' module by HECTD2 offers one explanation for the negative effect on anti-tumour immunity we observed in this study. However, the 'COX2' module is found only in a minority of SKCM samples (9.3\%), predominantly also expressing high levels of HECTD2 and lack evidence for T cell infiltration. In contrast, nearly half of SKCM samples expressed the 'HECTD2' module and were devoid of T cell infiltration, without expressing the 'COX2' module. These findings suggest that HECTD2 activity is responsible for COX2mediated immunosuppression, but may also activate more prevalent alternative immunosuppressive mechanisms, such as the balance of cytokines and chemokines. 
Using data from two separate studies [35, 36], we found that higher expression of HECTD2 in melanoma patients was predictive of a poor response to PD-1 blockade. Moreover, overexpression of HECTD2 in a mouse melanoma model diminished the effectiveness of the adaptive immune response to a model tumour antigen. These observations support an immunosuppressive role for HECTD2 in melanoma, which warrants further investigation. It is notable that the 'HECTD2' module is characterised by immune-related genes encoding predominantly transmembrane proteins, including CD47. By binding to its receptor SIRPa (signal receptor protein-alpha) on macrophages, CD47, ubiquitously expressed in healthy or transformed cells, inhibits their phagocytosis by macrophages, by sending 'do not eat me' signals [34, 38]. Elevated CD47 expression in cancer cells counteracts their pro-phagocytic signals and CD47 blockade is considered a valid target for immunotherapy [34, 38]. Collectively, these findings suggest an association of HECTD2 expression with multiple immune evasion pathways.

HECTD2 is widely expressed in healthy tissues, including the skin [18]. We find that this expression is maintained in primary melanoma and further elevated in metastatic melanoma [18], supporting a link between high HECTD2 expression and melanoma progression. However, several mechanisms that regulate HECTD2 expression and activity have been proposed, operating at distinct levels, as would be expected for genes involved in multiple cellular processes. A possible role for HECTD2 in melanoma was suggested by its regulation through the recently discovered [HECTD2-AS] $H E R V H-2$ antisense transcript expressed highly specifically in melanoma [18]. Antisense transcription has long been recognised as an effective regulator of gene activity [39] and appears to be the predominant form of HECTD2 regulation in human melanoma [18]. Other than melanoma, antisense [HECTD2-AS]HERVH transcripts have been detected in bladder adenocarcinoma, as well as healthy bladder and a few reproductive tissues [18]. In most healthy tissues and cancer types, however, HECTD2 expression is not subject to regulation by antisense transcription.

HECTD2 expression has also been shown to be directly regulated by miR-221 in prostate cancer cells [23]. MiR-221 and miR-222 are two small non-coding RNAs with the same seed sequence that have been implicated in cancer [40] and their targeting of HECTD2 could extend its involvement beyond melanoma. HECTD2 expression may additionally be regulated by the IncRNA ERP in human cancer, as has been suggested by studies in epithelial cells [41, 42].

Lastly, in addition to transcription and stability of the HECTD2 RNA, Coon et al. reported high frequency (8.5\%) of a singlenucleotide polymorphism affecting HECTD2 activity [22]. However, our independent analysis of all available genomes failed to detect this variant, excluding a contribution to the regulation of HECTD2 activity (Supplementary text).

Collectively, our results point to a critical role for HECTD2 in promoting melanoma cell-intrinsic proliferation and drug resistance and counteracting anti-tumour adaptive immunity and immunotherapy. Blocking this multifaceted melanoma-promoting function of HECTD2 may, therefore, be considered as a potential treatment of melanoma.

\section{METHODS}

Mice

Inbred C57BL/6J (B6) and severely immunodeficient $\mathrm{Rag}^{-1-} \| 12 \mathrm{rg}^{-/} \mathrm{Cd}_{47}{ }^{-1}$

mice were originally obtained from The Jackson Laboratory (Bar Harbor, $M E, U S A)$ and subsequently maintained at the Francis Crick Institute's animal facilities. Eight to twelve-week-old male and female gendermatched recipient mice were used for all experiments, randomly allocated to the different groups. All animal experiments were approved by the ethical committee of the Francis Crick Institute and conducted according to local guidelines and UK Home Office regulations under the Animals Scientific Procedures Act 1986 (ASPA).
Cell lines, transfection and transduction

$\mathrm{HCmel}$ cell lines $[29,32]$ and Braf ${ }^{\mathrm{V} 600 \mathrm{E}}$ cells [31] have been previously described. Detailed information about cell lines, transfection and transduction are available in Supplementary materials and methods.

\section{Statistical analysis}

Statistical comparisons were made using SigmaPlot 13.0 (Systat Software Inc., Germany). Parametric comparisons of normally distributed values that satisfied the variance criteria were made by unpaired Student's $t$-tests or One Way Analysis of variance (ANOVA) tests. Data that did not pass the variance test were compared with non-parametric two-tailed Mann-Whitney Rank Sum tests or ANOVA on Ranks tests.

Detailed information about the materials and methods used in the present study is available in Supplementary materials and methods.

\section{REFERENCES}

1. Sperka T, Wang J, Rudolph KL. DNA damage checkpoints in stem cells, ageing and cancer. Nat Rev Mol Cell Biol. 2012;13:579-90.

2. Wu S, Zhu W, Thompson P, Hannun YA. Evaluating intrinsic and non-intrinsic cancer risk factors. Nat Commun. 2018;9:3490.

3. Fridman WH, Pagès $F$, Sautès-Fridman $C$, Galon J. The immune contexture in human tumours: impact on clinical outcome. Nat Rev Cancer. 2012;12:298-306.

4. Gajewski TF, Schreiber H, Fu YX. Innate and adaptive immune cells in the tumor microenvironment. Nat Immunol. 2013;14:1014-22.

5. Hinshaw DC, Shevde LA. The tumor microenvironment innately modulates cancer progression. Cancer Res. 2019;79:4557-66.

6. Lau AN, Vander Heiden MG. Metabolism in the tumor microenvironment. Annu Rev Cancer Biol. 2020;4:17-40.

7. Bedognetti D, Hendrickx W, Ceccarelli M, Miller LD, Seliger B. Disentangling the relationship between tumor genetic programs and immune responsiveness. Curr Opin Immunol. 2016:39:150-8.

8. Spranger S, Gajewski TF. Impact of oncogenic pathways on evasion of antitumour immune responses. Nat Rev Cancer. 2018;18:139-47.

9. Wellenstein MD, de Visser KE. Cancer-cell-intrinsic mechanisms shaping the tumor immune landscape. Immunity. 2018;48:399-416.

10. Schumacher TN, Scheper W, Kvistborg P. Cancer neoantigens. Annu Rev Immunol. 2019;37:173-200.

11. Kassiotis G, Stoye JP. Immune responses to endogenous retroelements: taking the bad with the good. Nat Rev Immunol. 2016;16:207-19.

12. Ishak CA, De Carvalho DD. Reactivation of endogenous retroelements in cancer development and therapy. Annu Rev Cancer Biol. 2020;4:159-76.

13. Gentles AJ, Newman AM, Liu CL, Bratman SV, Feng W, Kim D, et al. The prognostic landscape of genes and infiltrating immune cells across human cancers. Nat Med. 2015;21:938-45.

14. MacKie RM, Hauschild A, Eggermont AM. Epidemiology of invasive cutaneous melanoma. Ann Oncol. 2009;20:vi1-7.

15. Alexandrov LB, Nik-Zainal S, Wedge DC, Aparicio SA, Behjati S, Biankin AV, et al. Signatures of mutational processes in human cancer. Nature. 2013;500:415-21.

16. Vesely MD, Kershaw MH, Schreiber RD, Smyth MJ. Natural innate and adaptive immunity to cancer. Annu Rev Immunol. 2011;29:235-71.

17. Pardoll DM. The blockade of immune checkpoints in cancer immunotherapy. Nat Rev Cancer. 2012;12:252-64.

18. Attig J, Young GR, Hosie L, Perkins D, Encheva-Yokoya V, Stoye JP, et al. LTR retroelement expansion of the human cancer transcriptome and immunopeptidome revealed by de novo transcript assembly. Genome Res. 2019;29:1578-90.

19. Wang Y, Argiles-Castillo D, Kane El, Zhou A, Spratt DE HECT E3 ubiquitin ligasesemerging insights into their biological roles and disease relevance. J Cell Sci. 2020;133:jcs228072.

20. Lloyd SE, Maytham EG, Pota H, Grizenkova J, Molou E, Uphill J, et al. HECTD2 is associated with susceptibility to mouse and human prion disease. PLoS Genet. 2009;5:e1000383.

21. Lloyd SE, Rossor M, Fox N, Mead S, Collinge J. HECTD2, a candidate susceptibility gene for Alzheimer's disease on 10q. BMC Med Genet. 2009;10:90.

22. Coon TA, McKelvey AC, Lear T, Rajbhandari S, Dunn SR, Connelly W, et al. The proinflammatory role of HECTD2 in innate immunity and experimental lung injury. Sci Transl Med. 2015;7:295ra109.

23. Sun T, Wang X, He HH, Sweeney CJ, Liu SX, Brown M, et al. MiR-221 promotes the development of androgen independence in prostate cancer cells via downregulation of HECTD2 and RAB1A. Oncogene. 2014;33:2790-800.

24. Suo C, Deng W, Vu TN, Li M, Shi L, Pawitan Y. Accumulation of potential driver genes with genomic alterations predicts survival of high-risk neuroblastoma patients. Biol Direct. 2018;13:14. 
25. The Cancer Genome Atlas Network. Genomic classification of cutaneous melanoma. Cell. 2015;161:1681-96.

26. Thorsson V, Gibbs DL, Brown SD, Wolf D, Bortone DS, Ou Yang TH, et al. The immune landscape of cancer. Immunity. 2018;48:812-30.e14.

27. Barretina J, Caponigro G, Stransky N, Venkatesan K, Margolin AA, Kim S, et al. The cancer cell line encyclopedia enables predictive modelling of anticancer drug sensitivity. Nature. 2012;483:603-07.

28. Tsherniak A, Vazquez F, Montgomery PG, Weir BA, Kryukov G, Cowley GS, et al. Defining a cancer dependency map. Cell. 2017;170:564-76.e16.

29. Bald T, Quast T, Landsberg J, Rogava M, Glodde N, Lopez-Ramos D, et al. Ultraviolet-radiation-induced inflammation promotes angiotropism and metastasis in melanoma. Nature. 2014;507:109-13.

30. Ottina E, Levy P, Eksmond U, Merkenschlager J, Young GR, Roels J, et al. Restoration of endogenous retrovirus infectivity impacts mouse cancer models. Cancer Immunol Res. 2018;6:1292-300.

31. Dhomen N, Reis-Filho JS, da Rocha Dias S, Hayward R, Savage K, Delmas V, et al. Oncogenic Braf induces melanocyte senescence and melanoma in mice. Cancer Cell. 2009;15:294-303.

32. Landsberg J, Kohlmeyer J, Renn M, Bald T, Rogava M, Cron M, et al. Melanomas resist T-cell therapy through inflammation-induced reversible dedifferentiation. Nature. 2012;490:412-6.

33. Zelenay S, van der Veen AG, Böttcher JP, Snelgrove KJ, Rogers N, Acton SE, et al. Cyclooxygenase-dependent tumor growth through evasion of immunity. Cell. 2015;162:1257-70.

34. Barclay AN, van den Berg TK. The interaction between signal regulatory protein alpha (SIRPa) and CD47: structure, function, and therapeutic target. Annu Rev Immunol. 2014;32:25-50.

35. Hugo W, Zaretsky JM, Sun L, Song C, Moreno BH, Hu-Lieskovan S, et al. Genomic and transcriptomic features of response to Anti-PD-1 therapy in metastatic melanoma. Cell. 2016;165:35-44.

36. Riaz N, Havel JJ, Makarov V, Desrichard A, Urba WJ, Sims JS, et al. Tumor and microenvironment evolution during immunotherapy with Nivolumab. Cell. 2017;171:934-49.e16.

37. Danelli L, Cornish G, Merkenschlager J, Kassiotis G. Default polyfunctional T helper 1 response to ample signal 1 alone. Cell Mol Immunol. 2020;1-14. https:// doi.org/10.1038/s41423-020-0415-x.

38. Takimoto $\mathrm{CH}$, Chao MP, Gibbs C, McCamish MA, Liu J, Chen JY, et al. The Macrophage 'Do not eat me' signal, CD47, is a clinically validated cancer immunotherapy target. Ann Oncol. 2019;30:486-89.

39. Pelechano V, Steinmetz LM. Gene regulation by antisense transcription. Nat Rev Genet. 2013;14:880-93.

40. Garofalo M, Quintavalle C, Romano G, Croce CM, Condorelli G. miR221/222 in cancer: their role in tumor progression and response to therapy. Curr Mol Med. 2012;12:27-33.

41. Rossi M, Bucci G, Rizzotto D, Bordo D, Marzi MJ, Puppo $M$, et al. LncRNA EPR controls epithelial proliferation by coordinating Cdkn1a transcription and mRNA decay response to TGF- $\beta$. Nat Commun. 2019;10:1969.

42. Zapparoli E, Briata P, Rossi M, Brondolo L, Bucci G, Gherzi R. Comprehensive multi-omics analysis uncovers a group of TGF- $\beta$-regulated genes among IncRNA EPR direct transcriptional targets. Nucleic Acids Res. 2020;48:9053-66.

\section{ACKNOWLEDGEMENTS}

We are grateful for assistance from the Advanced Light Microscopy, Biological Research Facility, Cell Services, Flow Cytometry and Scientific Computing facilities at the Francis Crick Institute. The results shown here are in whole or part based upon data generated by the TCGA Research Network (http://cancergenome.nih.gov). This work benefited from data assembled by the CCLE consortium. This work was supported by the Francis Crick Institute (FC001099, FC001169, FC001202), which receives its core funding from Cancer Research UK (CRUK), the UK Medical Research Council (MRC), and the Wellcome Trust; and by the Wellcome Trust (102898/B/13/Z to GK) and CRUK (C55533/A22158 to GK). This research was funded in whole, or in part, by the Wellcome Trust (102898/B/13/Z; FC001099, FC001169, FC001202). For the purpose of Open Access, the author has applied a CC BY public copyright licence to any Author Accepted Manuscript version arising from this submission.

\section{COMPETING INTERESTS}

GK is a scientific co-founder of EnaraBio and a member of its scientific advisory board. GK and GRY are consulting for EnaraBio. CS acknowledges grant support from Pfizer, AstraZeneca, Bristol Myers Squibb, Roche-Ventana, Boehringer-Ingelheim, Archer Dx Inc (collaboration in minimal residual disease sequencing technologies) and Ono Pharmaceutical, is an AstraZeneca Advisory Board member and Chief Investigator for the MeRmaiD1 clinical trial, has consulted for Pfizer, Novartis, GlaxoSmithKline, MSD, Bristol Myers Squibb, Celgene, AstraZeneca, Illumina, Genentech, Roche-Ventana, GRAIL, Medicxi, Bicycle Therapeutics, and the Sarah Cannon Research Institute, has stock options in Apogen Biotechnologies, Epic Bioscience, GRAIL, and has stock options and is co-founder of Achilles Therapeutics.

\section{ADDITIONAL INFORMATION}

Supplementary information The online version contains supplementary material available at https://doi.org/10.1038/s41388-021-01885-4.

Correspondence and requests for materials should be addressed to G.K.

Reprints and permission information is available at http://www.nature.com/ reprints

Publisher's note Springer Nature remains neutral with regard to jurisdictional claims in published maps and institutional affiliations.

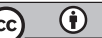

Open Access This article is licensed under a Creative Commons Attribution 4.0 International License, which permits use, sharing, adaptation, distribution and reproduction in any medium or format, as long as you give appropriate credit to the original author(s) and the source, provide a link to the Creative Commons license, and indicate if changes were made. The images or other third party material in this article are included in the article's Creative Commons license, unless indicated otherwise in a credit line to the material. If material is not included in the article's Creative Commons license and your intended use is not permitted by statutory regulation or exceeds the permitted use, you will need to obtain permission directly from the copyright holder. To view a copy of this license, visit http://creativecommons. org/licenses/by/4.0/.

(c) The Author(s) 2021, corrected publication 2021 